\title{
Cardiopulmonary resuscitation in a district general hospital: increased success over 7 years
}

R. D. THOMAS, J. H. WAITES, W. N. HUBBARD \& M. WICKS

Department of Cardiology, Royal United Hospital, Bath and Adelaide

\section{SUMMARY}

In a 12-month prospective survey of CPR (cardiopulmonary resuscitation), 32 out of 192 patients $(16 \cdot 6 \%)$ survived to go home. This is a clear improvement compared with 7 years previously. This is attributed to better training in the use and management of CPR and more widespread availability of defibrillators. Certain patients could not be resuscitated - those with electromechanical dissociation carcinoma, or multiple pathology. Age by itself was not a bar to resuscitation.

There is still a high rate of inappropriate calls, often because of uncertainty by nurses about the use of CPR. This could be improved with clearer guidelines in hospitals about the value of CPR in selected patients.

\section{INTRODUCTION}

Cardiopulmonary resuscitation (CPR) in hospitals has a low success rate with an average of $13 \%$ in the 11 studies reported by Woog \& Torzillo (1987). There are several reasons for this including the nature of the underlying disease, the effectiveness of the cardiac arrest team and the proportion of inappropriate calls. The latter include calls when it can be confidently predicted beforehand that resuscitation will fail.

Results from the Royal United Hospital were poor when studied prospectively in 1981 and reported briefly (Ridley \& Thomas 1982). There have been changes since then and in order to determine the present efficacy we studied prospectively all cardiac arrests.

Correspondence: $\operatorname{Dr}$ R. D. Thomas, Department of Cardiology, Royal United Hospital, Combe Park, Bath BA1 3NG, U.K. 


\section{METHODS}

All adult cardiac arrest calls, excluding those in the Coronary Care and Intensive Care Units, were documented for a 12-month period (1/7/87-1/7/88) in this 800 bedded District General Hospital. For the purpose of the study cardiac arrest was defined as any event that precipitated calling the cardiac arrest team. Basic information obtained at the arrest was recorded on a card, and completeness of the survey checked through records kept by the hospital switchboard. Suitability for resuscitation was determined at the time of the arrest using criteria similar to those of Hershey \& Fisher (1982).

The cardiac arrest team consisted of a medical registrar or SHO (who filled in the card), a pre-registration house physician, an anaesthetic registrar or $\mathrm{SHO}$ and a senior nurse. Standard basic and advanced life support methods were used.

\section{RESULTS}

Two hundred and twenty-six CPR calls were made, summarized in Figure 1. Thirty-four patients had clearly not arrested and 48 cases were considered inap-

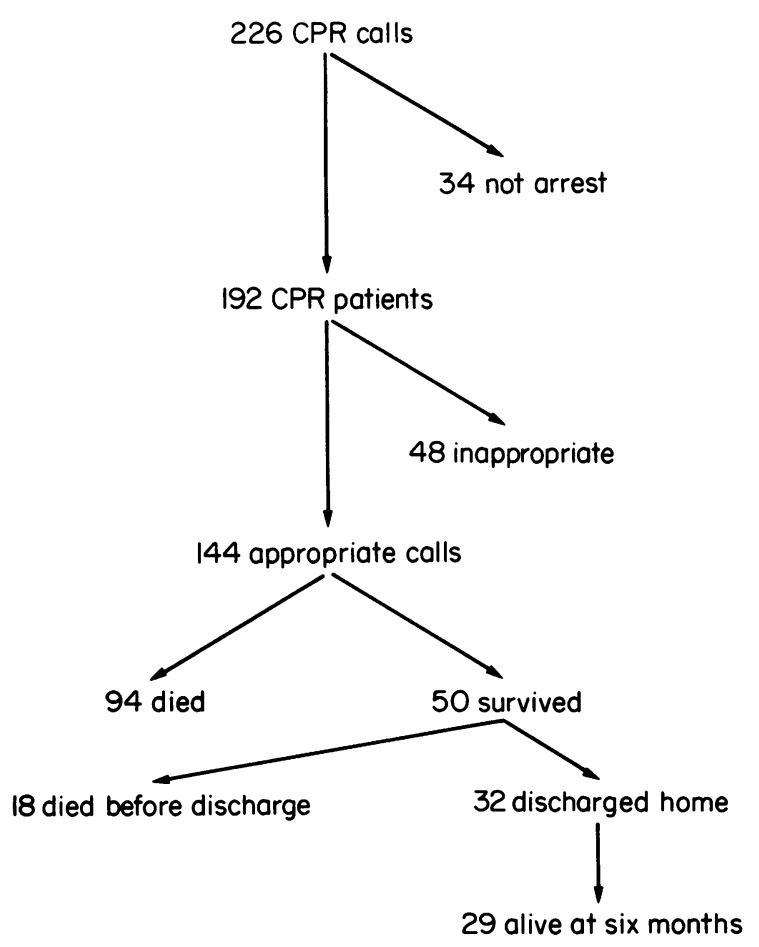

Fig. 1. Breakdown of CPR calls that were made 
propriate calls. Out of 144 appropriate calls 50 patients survived the arrest and of these 32 were discharged home, giving a success rate of 32/192 (16.6\%). Twentynine patients were alive at 6 months.

The highest success rate was in respiratory arrest with 8 out of 9 patients surviving. When the arrest was primarily cardiac, 14 out of 39 patients survived ventricular tachycardia or fibrillation and 8 out of 67 survived asystole. No patients survived electromechanical dissociation.

Analysis of the site of calls is shown in Table 1. The Accident \& Emergency Department had the highest rate of success and number of inappropriate calls. Six of the 10 survivors had taken an overdose and four had acute myocardial infarction. The age distribution of appropriate calls is shown in Figure 2. The high rate of success in patients aged $11-30$ is because these were predominantly respiratory arrests in patients with drug overdose. There were no other differences with age and three out of 13 patients over the age of 80 years survived an arrest. The commonest underlying pathology was ischaemic heart disease (Table 2). There was no success in patients with known carcinoma, or with a post-operative cardiac arrest.

Nearly $40 \%$ of the cardiac arrest calls were for patients who had not had a cardiac arrest or for whom the call was clearly inappropriate. The non-arrests were

Table 1. Results of CPR related to site of calls (Total Numbers).

\begin{tabular}{lccr}
\hline Site & Appropriate & Inappropriate & Surviving \\
\hline Medical ward & 99 & 26 & 20 \\
Surgical ward & 12 & 4 & 2 \\
A \& E Department & 28 & 15 & 10 \\
Other & 4 & 0 & 0 \\
\hline
\end{tabular}

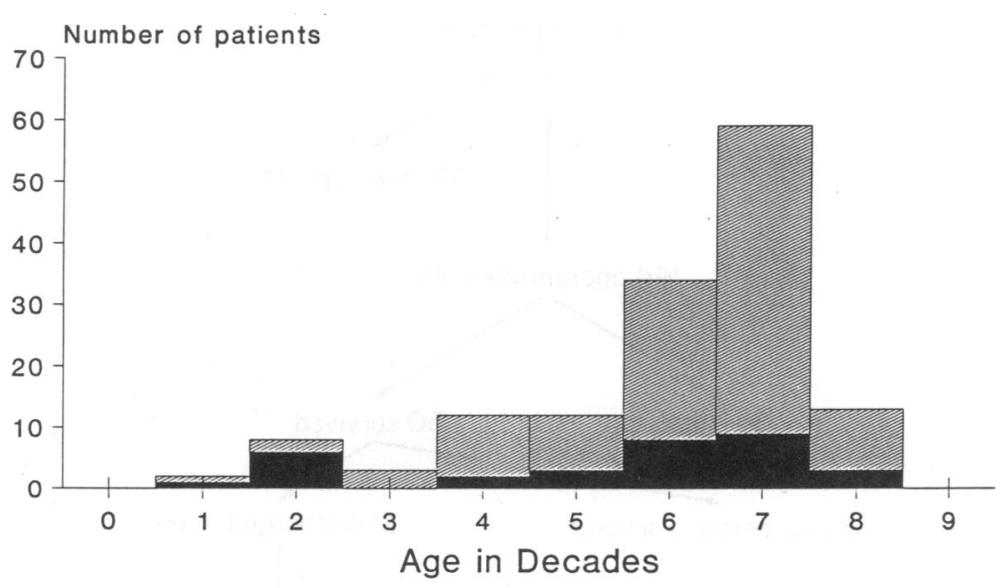

Fig. 2. Age distribution. 
Table 2. Mechanism of cardiac arrest

\begin{tabular}{lcc}
\hline Mechanism of arrest & Total number & Number surviving \\
\hline VF/VT & 39 & 14 \\
Asystole & 67 & 8 \\
EMD & 23 & 0 \\
Unknown & 3 & 0 \\
Respiratory & 9 & 8 \\
Shock & 2 & 2 \\
\hline EMD = Electromechanical dissociation.
\end{tabular}

Table 3. Underlying disease in appropriate calls.

\begin{tabular}{lrc}
\hline Diagnosis & No. & No. surviving \\
\hline IHD/LVF & 82 & 12 \\
Resp. Failure & 9 & 2 \\
Overdose & 10 & 7 \\
C.V.A. & 8 & 2 \\
Carcinoma & 5 & 0 \\
Post-op & 5 & 0 \\
Pulm. Embolism & 4 & 1 \\
R.T.A. & 4 & 1 \\
Conduction Dis. & 3 & 3 \\
Septicaemia & 3 & 1 \\
Aortic Aneurysm & 6 & 2 \\
Other & 5 & 1 \\
\hline
\end{tabular}

Table 4. Reason for inappropriate calls.

\begin{tabular}{lr}
\hline Multiple pathology with poor prognosis & 14 \\
Malignant disease & 10 \\
End-stage cardiac disease & 10 \\
Brought in dead & 6 \\
Age & 4 \\
Not for CPR & 4 \\
\hline
\end{tabular}

usually either vasovagal syncope or sleep. Calls were considered inappropriate for several reasons, shown in Table 4 . There was no association between inappropriate calls and time of day or site of arrest but two-thirds were over 70-years-old.

A comparison of the results with those in 1981 is shown in Figure 3. The increased number of calls can be seen (from 99 to 226) with an increased success rate from two out of 99 to 32 out of 192. This has been accompanied by an increased number of inappropriate calls. In 1981 there were 760 beds on the hospital site and the total number of hospital deaths was approximately the same as $1987 / 88$. 


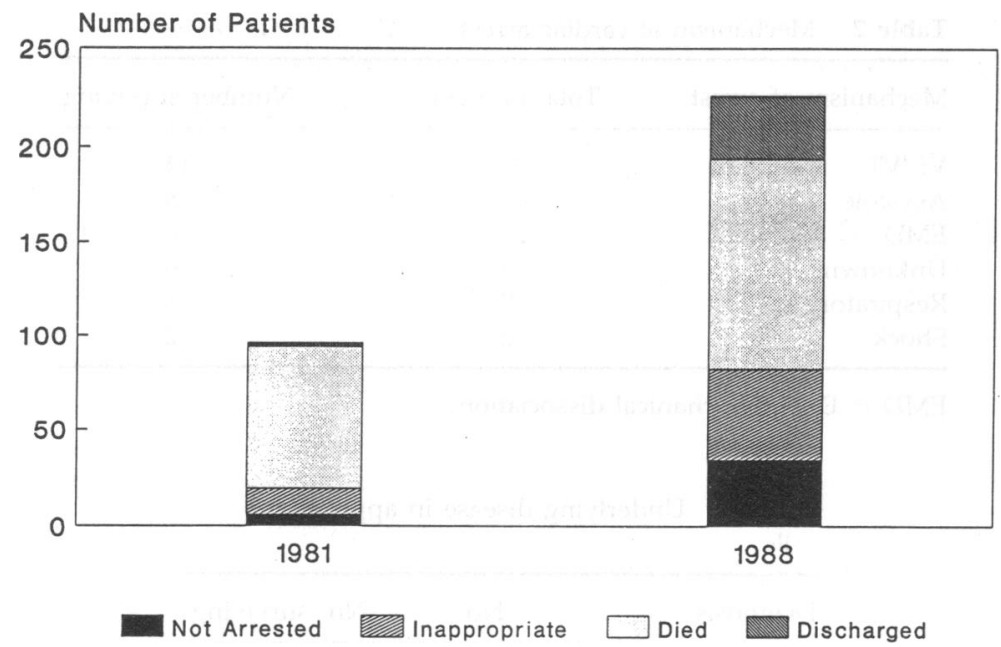

Fig. 3. Comparison of CPR calls between 1981 and 1988.

\section{DISCUSSION}

Most similar reports have included coronary care units (CCUs). We purposefull $\mathbb{B} \vec{\circ}$ excluded these because success rates in patients with acute myocardial infarction are known to be. Recalculating the figures from Woog \& Torzillo (1987) for puble cations later than 1970 to exclude arrests in CCUs gives a success rate of less than $9 \%-$ and as low as 3\% in one series (Hershey \& Fisher, 1982).

We have found a clear increase in the number of CPR calls between 1981 and 1988 , both appropriate and inappropriate. There has also been an increase in success rate. We think that the latter is the result of better training for junior medical staff, having recognized problems in this area. In addition there has been an increased distribution of defibrillators throughout the hospital from one per six wards in 1981 to one per two wards at present. However, there has also been an increase in the number of inappropriate calls. An important reason for this is increasing concern by nursing staff about possible criticism if patients are allowed to die without resuscitation. The Royal College of Nursing Code of Practice states that in cases of unexpected cardiac and respiratory arrest nursing staff have a responsibility to initiate resuscitative measures. Consequently nurses frequently face a dilemma about the need for resuscitation (Thom 1988).

Twenty-five percent of the calls in this hospital were inappropriate, using information available from the arrest team at the time. We found later that a significant proportion of calls thought initially to be appropriate were in fact inappropriate, but this cannot always be appreciated by the arrest team at the time of the call. This particularly applies to the Accident and Emergency Department where a higher proportion of inappropriate calls may be acceptable.

Our study confirms the work of others that patients with malignancy, respiratory 
failure, or severe cardiac failure have a very low chance of survival, but age per se is not a bar to resuscitation. This contradicts a recent study which found that patients over the age of 70 years often survived initial resuscitation, but did not live to be discharged (Taffet et al., 1988).

We are certain that there should be clearer guidelines about which patients are not suitable for CPR - patients in whom it can predicted confidently that CPR will fail. A high proportion of inappropriate calls is demoralizing to the arrest team and detracts from the enormous benefit of CPR to selected patient groups.

\section{REFERENCES}

Her̀shey C. O. \& Fisher L. (1982) Why outcome of cardiopulmonary resuscitation in general wards is poor. Lancet 1, 31-4.

Ridley M. \& Thomas R. D. (1982) Cardiopulmonary resuscitation in general wards. Lancet 1, 20.

Taffet G. E., Teasdale T. A. \& Luchi R. J. (1988) In-hospital cardiopulmonary resuscitation. Journal of American Medical Association 260, 2069-72.

Thom A. (1988) Who decides? Nursing Times 85, 35-7.

Woog R. H. \& Torzillo P. J. (1987) In-hospital cardiopulmonary resuscitation: prospective survey of management and outcome. Anaesthesia and Intensive Care 15, 193-8. 